\title{
Weakly coupled Schrödinger operators on regular metric trees
}

\author{
Hynek Kovařík * \\ Institute of Analysis, Dynamics and Modeling, Universität Stuttgart, \\ PF 8011 40, D-70569 Stuttgart, Germany. \\ E-mail: kovarik@mathematik.uni-stuttgart.de
}

\begin{abstract}
Spectral properties of the Schrödinger operator $A_{\lambda}=-\Delta+\lambda V$ on regular metric trees are studied. It is shown that as $\lambda$ goes to zero the behavior of the negative eigenvalues of $A_{\lambda}$ depends on the global structure of the tree.
\end{abstract}

Mathematics Subject Classification: 34L40, 34B24, 34B45.

Key words: Schrödinger operator, regular metric trees, weak coupling.

\section{Introduction}

A rooted metric tree $\Gamma$ consists of the set of vertices and the set of edges, i.e. one dimensional intervals connecting the vertices, see section 2 for details. A Schrödinger operator of the type

$$
A_{\lambda}=-\Delta+\lambda V, \quad \lambda>0, \quad \text { in } \quad L^{2}(\Gamma)
$$

is usually defined on a set of functions which satisfy the so-called Kirchhoff boundary conditions at the vertices of the tree, see (2), and a suitable condition at its root, which ensures the self-adjointness of $A_{\lambda}$. Below we shall always assume that $V$ is symmetric which means that it depends only on the distance from the root of $\Gamma$.

Spectral theory of such operators has recently attracted a considerable attention, 4, 5, 6, 7, 10, 11. In [10] a detailed asymptotic analysis of the counting function of the discrete eigenvalues of $A_{\lambda}$ in the limit $\lambda \rightarrow \infty$ was done for a special class of regular trees whose edges have a constant length. It was shown, see [10, that depending on the decay of $V$ this asymptotics is either of the Weyl type or it is fully determined by the behavior of $V$ at infinity.

\footnotetext{
*Also on the leave from Nuclear Physics Institute, Academy of Sciences, 25068 Řež near
} Prague, Czech Republic. 
In this paper we are interested in the spectral behavior of $A_{\lambda}$ in the weak coupling when $\lambda \rightarrow 0$. The intuitive expectation is that the weak coupling behavior of $A_{\lambda}$ should depend on the rate of the growth of the tree $\Gamma$. This is motivated by the fact that the weak coupling properties of the operator $A_{\lambda}$ in $L^{2}\left(\mathbb{R}^{n}\right)$ depend strongly on $n$. In particular, it is well known, see [11, that for $n=1,2$ the negative eigenvalues of $A_{\lambda}$ appear for any $\lambda>0$, provided $\int_{\mathbb{R}^{n}} V<0$, while for $n \geq 3$ the negative spectrum of $A_{\lambda}$ remains empty for $\lambda$ small enough. Moreover, for $\varepsilon(\lambda)$, the lowest eigenvalue of $A_{\lambda}$, the following asymptotic formulae hold true, [1]:

For $n=1$ :

$$
\varepsilon(\lambda) \sim-\lambda^{2}, \quad \lambda \rightarrow 0 .
$$

For $n=2$ :

$$
\varepsilon(\lambda) \sim-e^{-\lambda^{-1}}, \quad \lambda \rightarrow 0 .
$$

In our model, we assign to the tree $\Gamma$ a so-called global dimension $d$, see Definition 2 below. Roughly speaking, it tells us how fast the number of the branches of $\Gamma$ grows as a function of the distance from the root. If the latter grows with the power $d-1$ at infinity, then we say that $d$ is the global dimension of the tree. We use the notation global in order to distinguish $d$ from the local dimension of the tree, which is of course one. Since $d$ can be in general any real number larger or equal to one, it is natural to ask how the weak coupling behavior looks like for non-integer values of $d$ and what is the condition on $V$ under which the eigenvalues appear. We will show, see section $[5$ that if $d \in[1,2]$ and $\int_{\Gamma} V<0$, then $A_{\lambda}$ possesses at least one negative eigenvalue for any $\lambda>0$ and for $\lambda$ small enough this eigenvalue is unique and satisfies

$$
c_{1} \lambda^{\frac{2}{2-d}} \leq|\varepsilon(\lambda)| \leq c_{2} \lambda^{\frac{2}{2-d}}, \quad 1 \leq d<2,
$$

for some suitable constants $c_{1}, c_{2}$. As expected, the power diverges as $d$ approaches 2 from the left. Notice, that our result qualitatively agrees with the precise asymptotic formula for $\varepsilon(\lambda)$ on branching graphs with one vertex and finitely many edges, which was found in [5]. Such graphs correspond to $d=1$ in our setting.

In order to study the operator $A_{\lambda}$ we make use of the decomposition (3), see Theorem [1] which was proved by Naimark and Solomyak in [6] 17, see also [4]. In section [3.1] we introduce certain auxiliary operators, whose eigenvalues will give us the estimate on $\varepsilon(\lambda)$ from above and from below. In order to establish (11) we find the asymptotics of the lowest eigenvalues of the auxiliary operators, which are of the same order. This is done in section 5.1 In section [5.3 it is shown, under some regularity conditions on $V$, that if the tree grows too fast, i.e. $d>2$, then $A_{\lambda}$ has no weakly coupled eigenvalues at all, that is the discrete spectrum remains empty for $\lambda$ small enough. Besides, in section 4 we give some estimates on the number of eigenvalues of the individual operators in the decomposition (3), which are used in the proofs of the main results, but might be of an independent interest as well. 
Throughout the text we will employ the notation $\alpha:=d-1$ and $\nu:=\frac{2-d}{2}$. For a real-valued function $f$ and a real non-integer number $\mu$ we will use the shorthand

$$
f^{\mu}:=\operatorname{sign} f|f|^{\mu}=\frac{f|f|^{\mu}}{|f|} .
$$

Finally, given a self-adjoint operator $T$ on a Hilbert space $\mathcal{H}$ we denote by $N_{-}(T ; s)$ the number of eigenvalues, taking into account their multiplicities, of $T$ on the left of the point $s$. For $s=0$ we will write $N_{-}(T)$ instead of $N_{-}(T ; 0)$.

\section{Preliminaries}

We define a metric tree $\Gamma$ with the root $o$ following the construction given in [6]. Let $\mathcal{V}(\Gamma)$ be the set of vertices and $\mathcal{E}(\Gamma)$ be the set of edges of $\Gamma$. The distance $\rho(y, z)$ between any two points $y, z \in \Gamma$ is defined in a natural way as the length of the unique path connecting $y$ and $z$. Consequently, $|y|$ is equal to $\rho(y, o)$. We write $y \preceq z$ if $y$ lies on the unique simple path connecting $o$ with $z$. For $y \preceq z$ we define

$$
<y, z>:=\{x \in \Gamma: y \preceq x \preceq z\} .
$$

If $e=\langle y, z>$ is an edge, then $y$ and $z$ are its endpoints. For any vertex $z$ its generation $\operatorname{Gen}(z)$ is defined by

$$
\operatorname{Gen}(z)=\#\{x \in \mathcal{V}: o \prec x \preceq z\} .
$$

The branching number $b(z)$ of the vertex $z$ is equal to the number of edges emanating from $z$. We assume that $b(z)>1$ for any $z \neq o$ and $b(o)=1$.

Definition 1. A tree $\Gamma$ is called regular if all the vertices of the same generation have equal branching numbers and all the edges of the same generation have equal length.

We denote by $t_{k}>0$ the distance between the root and the vertices of the $k-$ th generation and by $b_{k} \in \mathbb{N}$ their corresponding branching number. For each $k \in \mathbb{N}$ we define the so-called branching function $g_{k}: \mathbb{R}_{+} \rightarrow \mathbb{R}_{+}$by

$$
g_{k}(t):= \begin{cases}0 & \text { if } t<t_{k}, \\ 1 & \text { if } t_{k} \leq t \leq t_{k+1}, \\ b_{k+1} b_{k+2} \cdots b_{n} & \text { if } t_{n} \leq t \leq t_{n+1}, k<n,\end{cases}
$$

and

$$
g_{0}(t):=b_{0} b_{1} \cdots b_{n} \quad t_{n} \leq t \leq t_{n+1} .
$$

It follows directly from the definition that

$$
g_{0}(t)=\#\{x \in \Gamma:|x|=t\} .
$$

Obviously $g_{0}(\cdot)$ is a non-decreasing function and the rate of growth of $g_{0}$ determines the rate of growth of the tree $\Gamma$. In particular, if one denotes by $\Gamma(t):=\{x \in \Gamma:|x| \leq t\}$, the "ball" of radius $t$, then $g_{0}$ tells us how fast the surface of $\Gamma(t)$ grows with $t$. This motivates the following 
Definition 2. If there exist positive constants $a^{-}, a^{+}$and $T_{0}$, such that for all $t \geq T_{0}$ the inequalities

$$
a^{-} \leq \frac{g_{0}(t)}{t^{d-1}} \leq a^{+}
$$

hold true, then we say that $\mathrm{d}$ is the global dimension of the tree $\Gamma$.

We note that in the case of the so-called homogeneous metric trees treated in [10] the function $g_{0}(t)$ grows faster than any power of $t$. Formally, this corresponds to $d=\infty$ in the above definition. From now on we will work under the assumption that $d<\infty$.

\section{$3 \quad$ Schrödinger operators on $\Gamma$}

We will consider potential functions $V$ which satisfy the

Assumption A. $V: \mathbb{R}_{+} \rightarrow \mathbb{R}$ is measurable, bounded and $\lim _{t \rightarrow \infty} V(t)=0$.

For a given function $V$ which satisfies the Assumption $\mathrm{A}$ we define the Schrödinger operator $A_{\lambda}$ as the self-adjoint operator in $L^{2}(\Gamma)$ associated with the closed quadratic form

$$
Q_{\lambda}[u]:=\int_{\Gamma}\left(\left|u^{\prime}\right|^{2}+\lambda V(|x|)|u|^{2}\right) d x,
$$

with the form domain $D(Q)=H^{1}(\Gamma)$ consisting of all continuous functions $u$ such that $u \in H^{1}(e)$ on each edge $e \in \mathcal{E}(\Gamma)$ and

$$
\int_{\Gamma}\left(\left|u^{\prime}\right|^{2}+|u|^{2}\right) d x<\infty .
$$

The domain of $A_{\lambda}$ consists of all continuous functions $u$ such that $u^{\prime}(o)=0$, $u \in H^{2}(e)$ for each $e \in \mathcal{E}(\Gamma)$ and such that at each vertex $z \in \mathcal{V}(\Gamma) \backslash\{o\}$ the matching conditions

$$
u_{-}(z)=u_{1}(z)=\cdots=u_{b(z)}(z), \quad u_{1}^{\prime}(z)+\cdots+u_{b(z)}^{\prime}(z)=u_{-}^{\prime}(z)
$$

are satisfied, where $u_{-}$denotes the restriction of $u$ on the edge terminating in $z$ and $u_{j}, j=1, \ldots, b(z)$ denote respectively the restrictions of $u$ on the edges emanating from $z$, see [6] for details. Notice that $A_{\lambda}$ satisfies the Neumann boundary condition at the root $o$.

The following result by Naimark and Solomyak, see [6] 7], also established by Carlson in 4, makes it possible to reduce the spectral analysis of $A_{\lambda}$ to the analysis of one dimensional Schrödinger operators in weighted $L^{2}\left(\mathbb{R}_{+}\right)$spaces:

Theorem 1. Let $V$ be measurable and bounded and suppose that $\Gamma$ is regular. Then $A_{\lambda}$ is unitarily equivalent to the following orthogonal sum of operators:

$$
A_{\lambda} \sim A_{\lambda, 0} \oplus \sum_{k=1}^{\infty} \oplus A_{\lambda, k}^{\left[b_{1} \ldots b_{k-1}\left(b_{k}-1\right)\right]} .
$$


Here the symbol $A_{\lambda, k}^{\left[b_{1} \ldots b_{k-1}\left(b_{k}-1\right)\right]}$ means that the operator $A_{\lambda, k}$ enters the orthogonal sum $\left[b_{1} \ldots b_{k-1}\left(b_{k}-1\right)\right]$ times. For each $k \in \mathbb{N}$ the corresponding selfadjoint operator $A_{\lambda, k}$ acts in $L^{2}\left(\left(t_{k}, \infty\right), g_{k}\right)$ and is associated with the closed quadratic form

$$
Q_{k}[f]=\int_{t_{k}}^{\infty}\left(\left|f^{\prime}\right|^{2}+\lambda V(t)|f|^{2}\right) g_{k}(t) d t
$$

whose form domain is given by the the weighted Sobolev space $D\left(Q_{k}\right)=$ $H_{0}^{1}\left(\left(t_{k}, \infty\right), g_{k}\right)$ which consists of all functions $f$ such that

$$
\int_{t_{k}}^{\infty}\left(\left|f^{\prime}\right|^{2}+|f|^{2}\right) g_{k}(t) d t<\infty, \quad f\left(t_{k}\right)=0 .
$$

The operator $A_{\lambda, 0}$ acts in the weighted space $L^{2}\left(\mathbb{R}_{+}, g_{0}\right)$ and is associated with the closed form

$$
Q_{0}[f]=\int_{0}^{\infty}\left(\left|f^{\prime}\right|^{2}+\lambda V(t)|f|^{2}\right) g_{0}(t) d t
$$

with the form domain $D\left(Q_{0}\right)=H^{1}\left(\mathbb{R}_{+}, g_{0}\right)$ which consists of all functions $f$ such that

$$
\int_{0}^{\infty}\left(\left|f^{\prime}\right|^{2}+|f|^{2}\right) g_{0}(t) d t<\infty
$$

see also [1].

\subsection{Auxiliary operators}

Let $d$ be the global dimension of $\Gamma$. Definition (2) implies that for each $k \in \mathbb{N}_{0}$ there exist positive constants $a_{k}^{-}$and $a_{k}^{+}$, such that

$$
a_{k}^{-}(1+t)^{\alpha}=: g_{k}^{-}(t) \leq g_{k}(t) \leq g_{k}^{+}(t):=a_{k}^{+}(1+t)^{\alpha}, \quad t \in\left[t_{k}, \infty\right) .
$$

Now assume that the Rayleigh quotient

$$
\frac{\int_{t_{k}}^{\infty}\left(\left|f^{\prime}\right|^{2}+\lambda V(t)|f|^{2}\right) g_{k}(t) d t}{\int_{t_{k}}^{\infty}|f|^{2} g_{k}(t) d t}
$$

of the operator $A_{\lambda, k}, k \geq 0$ is negative for some $f \in D\left(Q_{k}\right)$. From (4) follows that

$$
\begin{aligned}
& \frac{\int_{t_{k}}^{\infty}\left(\left|f^{\prime}\right|^{2}+\lambda V_{k}^{-}(t)|f|^{2}\right)(1+t)^{\alpha} d t}{\int_{t_{k}}^{\infty}|f|^{2}(1+t)^{\alpha} d t} \leq \frac{\int_{t_{k}}^{\infty}\left(\left|f^{\prime}\right|^{2}+\lambda V(t)|f|^{2}\right) g_{k}(t) d t}{\int_{t_{k}}^{\infty}|f|^{2} g_{k}(t) d t} \\
& \leq \frac{\int_{t_{k}}^{\infty}\left(\left|f^{\prime}\right|^{2}+\lambda V_{k}^{+}(t)|f|^{2}\right)(1+t)^{\alpha} d t}{\int_{t_{k}}^{\infty}|f|^{2}(1+t)^{\alpha} d t}
\end{aligned}
$$

where

$$
V_{k}^{-}(t):=\frac{g_{k}(t)}{g_{k}^{-}(t)} V(t), \quad V_{k}^{+}(t):=\frac{g_{k}(t)}{g_{k}^{+}(t)} V(t) .
$$


It is thus natural to introduce the auxiliary operators $A_{\lambda, k}^{ \pm}$acting in the Hilbert space $L^{2}\left(\left(t_{k}, \infty\right),(1+t)^{\alpha}\right)$ and associated with the quadratic forms

$$
Q_{k}^{ \pm}[f]=\int_{t_{k}}^{\infty}\left(\left|f^{\prime}\right|^{2}+\lambda V_{k}^{ \pm}(t)|f|^{2}\right)(1+t)^{\alpha} d t, \quad f \in D\left(Q_{k}\right), k \in \mathbb{N}_{0} .
$$

The variational principle, see e.g. 3], and (5) thus imply that

$$
N_{-}\left(A_{\lambda, k}^{+} ; s\right) \leq N_{-}\left(A_{\lambda, k} ; s\right) \leq N_{-}\left(A_{\lambda, k}^{-} ; s\right), \quad s \leq 0, k \in \mathbb{N}_{0} .
$$

Let $E_{n, k}(\lambda)$ be the non-decreasing sequence of negative eigenvalues of the operators $A_{\lambda, k}$ and let $E_{n, k}^{ \pm}(\lambda)$ be the analogous sequences corresponding to the operators $A_{\lambda, k}^{ \pm}$respectively. In all these sequences each eigenvalue occurs according to its multiplicity. Relation (7) and variational principle then yield

$$
E_{n, k}^{-}(\lambda) \leq E_{n, k}(\lambda) \leq E_{n, k}^{+}(\lambda), \quad k \in \mathbb{N}_{0}, n \in \mathbb{N},
$$

and

$$
\inf \sigma_{e s s}\left(A_{\lambda, k}^{-}\right) \leq \inf \sigma_{e s s}\left(A_{\lambda, k}\right) \leq \inf \sigma_{e s s}\left(A_{\lambda, k}^{+}\right), \quad k \in \mathbb{N}_{0}
$$

Next we introduce the transformation $U$ by

$$
(U f)(t)=(1+t)^{\alpha / 2} f(t)=: \varphi(t),
$$

which maps $L^{2}\left(\left(t_{k}, \infty\right),(1+t)^{\alpha}\right)$ unitarily onto $L^{2}\left(\left(t_{k}, \infty\right)\right)$. We thus get

Lemma 1. Let $V$ satisfy the assumptions of Theorem [1] Then

(i) For each $k \in \mathbb{N}$ the operators $A_{\lambda, k}^{ \pm}$are unitarily equivalent to the selfadjoint operators $B_{\lambda, k}^{ \pm}$in $L^{2}\left(\left(t_{k}, \infty\right)\right)$, which act as

$$
\left(B_{\lambda, k}^{ \pm} \varphi\right)(t)=-\varphi^{\prime \prime}(t)+\frac{(d-1)(d-3)}{4(1+t)^{2}} \varphi(t)+\lambda V_{k}^{ \pm}(t) \varphi(t),
$$

and whose domains consist of all functions $\varphi \in H^{2}\left(\left(t_{k}, \infty\right)\right)$ such that

$$
\varphi\left(t_{k}\right)=0
$$

(ii) $A_{\lambda, 0}^{ \pm}$are unitarily equivalent to the self-adjoint operators $B_{\lambda, 0}^{ \pm}$in $L^{2}\left(\mathbb{R}_{+}\right)$, acting as

$$
\left(B_{\lambda, 0}^{ \pm} \varphi\right)(t)=-\varphi^{\prime \prime}(t)+\frac{(d-1)(d-3)}{4(1+t)^{2}} \varphi(t)+\lambda V_{0}^{ \pm}(t) \varphi(t),
$$

with the domain that consists of all $\varphi \in H^{2}\left(\mathbb{R}_{+}\right)$such that

$$
\varphi^{\prime}(0)=\frac{d-1}{2} \varphi(0) .
$$


Proof. For each $k \in \mathbb{N}_{0}$ we have

$$
B_{\lambda, k}^{ \pm}=U A_{\lambda, k}^{ \pm} U^{-1}, \quad\|f\|_{L^{2}\left(\left(t_{k}, \infty\right),(1+t)^{\alpha}\right)}=\|U f\|_{L^{2}\left(\left(t_{k}, \infty\right)\right)} .
$$

The statement of the Lemma then follows by a direct calculation keeping in mind that the functions $f$ from the domain of the operators $A_{\lambda, 0}^{ \pm}$satisfy $f^{\prime}(0)=0$.

Remark 1. If $V$ satisfies assumption A then the inequalities (9) and standard arguments from the spectral theory of Schrödinger operators, see e.g. 8, Chap.13.4], imply that

$$
\inf \sigma_{\text {ess }}\left(A_{\lambda, k}^{-}\right)=\inf \sigma_{\text {ess }}\left(A_{\lambda, k}\right)=\inf \sigma_{\text {ess }}\left(A_{\lambda, k}^{+}\right)=0, \quad \forall k \in \mathbb{N}_{0} .
$$

Moreover, constructing suitable Weyl sequences for the operators $A_{\lambda, k}$ in the similar way as it was done in [11] for the Laplace operator, one can actually show that

$$
\sigma_{\text {ess }}\left(A_{\lambda, k}\right)=[0, \infty), \quad \forall k \in \mathbb{N}_{0} .
$$

\section{$4 \quad$ Number of bound states}

From Theorem 1 and equation (13) we can see that if $V$ satisfies assumption $A$ then

$$
\sigma_{\text {ess }}\left(A_{\lambda}\right)=[0, \infty) .
$$

In order to analyze the discrete spectrum of $A_{\lambda}$ we first study the number of bound states of the individual operators in the decomposition (3).

We start by proving an auxiliary Proposition. Given a real valued measurable bounded function $\tilde{V}$ we consider the self-adjoint operator $\tilde{B}_{\lambda}$ acting in $L^{2}\left(\mathbb{R}_{+}\right)$as

$$
\left(\tilde{B}_{\lambda} \varphi\right)(t)=-\varphi^{\prime \prime}(t)+\frac{(d-1)(d-3)}{4 t^{2}} \varphi(t)+\lambda \tilde{V}(t) \varphi(t),
$$

and whose domain consist of all functions $\varphi \in H^{2}\left(\mathbb{R}_{+}\right)$such that $\varphi(0)=0$. We have

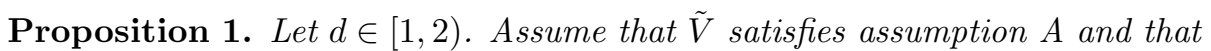
$\int_{0}^{\infty} t|\tilde{V}(t)| d t<\infty$. Then

$$
N_{-}\left(\tilde{B}_{\lambda}\right) \leq \lambda \tilde{K}(d) \int_{0}^{\infty} t|\tilde{V}(t)| d t
$$

where

$$
\tilde{K}(d)=\frac{\pi}{2 \sin (\nu \pi) \Gamma(1-\nu) \Gamma(1+\nu)} .
$$


Proof. We write

$$
\tilde{B}_{\lambda, 0}=\tilde{B}_{0}+\lambda \tilde{V}, \quad \tilde{B}_{0}:=-\frac{d^{2}}{d t^{2}}+\frac{(d-1)(d-3)}{4 t^{2}} .
$$

Moreover, without loss of generality we may assume that $\tilde{V}<0$.

By the Birman-Schwinger principle, see e.g. [3], the number of eigenvalues of $\tilde{B}_{\lambda}$ to the left of the point $-\kappa^{2}$ then does not exceed the trace of the operator

$$
\lambda|\tilde{V}|^{1 / 2}\left(\tilde{B}_{0}+\kappa^{2}\right)^{-1}|\tilde{V}|^{1 / 2} .
$$

The integral kernel $\tilde{G}\left(t, t^{\prime}, \kappa\right)$ of the operator $\left(\tilde{B}_{0}+\kappa^{2}\right)^{-1}$ can be calculated by using the Sturm-Liouville theory. We get

$$
\tilde{G}\left(t, t^{\prime}, \kappa\right)=\left\{\begin{array}{cc}
\frac{\pi i}{4} v_{1}(t, \kappa) v_{2}\left(t^{\prime}, \kappa\right) & t \geq t^{\prime} \\
\frac{\pi i}{4} v_{1}\left(t^{\prime}, \kappa\right) v_{2}(t, \kappa) & t<t^{\prime}
\end{array},\right.
$$

with

$$
\begin{aligned}
& v_{1}(t, \kappa)=\sqrt{t} H_{\nu}^{(1)}(i \kappa t), \\
& v_{2}(t, \kappa)=\sqrt{t} H_{\nu}^{(1)}(i \kappa t)+\sqrt{t} H_{\nu}^{(2)}(i \kappa t),
\end{aligned}
$$

where $H_{\nu}^{(1)}$ resp. $H_{\nu}^{(2)}$ denote Hankel's functions of the first resp. second kind, see e.g. [12. Since $\int_{0}^{\infty} t|\tilde{V}(t)| d t<\infty$, we can pass to the limit $\kappa \rightarrow 0$ in the corresponding integral, using the Lebesgue dominated convergence theorem, and calculate the trace to get

$$
N_{-}\left(\tilde{B}_{\lambda}\right) \leq \lambda \int_{0}^{\infty}|\tilde{V}(t)||\tilde{G}(t, t, 0)| d t=\lambda \tilde{K}(d) \int_{0}^{\infty} t|\tilde{V}(t)| d t .
$$

Here we have used the fact that $\tilde{G}(t, t, \kappa) \rightarrow t \tilde{K}(d)$ pointwise as $\kappa \rightarrow 0$, which follows from the asymptotic behavior of the Hankel functions at zero, see e.g. [1.

Remark 2. For $d=1$ we have $\tilde{K}(1)=1$ and (16) gives the well known Bargmann inequality, 2]. On the other hand, $\tilde{K}(d)$ diverges as $d \rightarrow 2-$. This is expected because the operator $-\frac{d^{2}}{d t^{2}}-\frac{1}{4 t^{2}}+\lambda V$ with Dirichlet b.c. at zero does have at least one negative eigenvalue for any $\lambda>0$ if the integral of $V$ is negative.

Armed with Proposition 1 we can prove

Corollary 1. Let $1 \leq d<2$. Assume that $V$ satisfies assumption $\square$ and that $\int_{0}^{\infty} t|V(t)| d t<\infty$. Then

$$
N_{-}\left(A_{\lambda, 0}\right) \leq 1+\lambda K(d) \int_{0}^{\infty}|V(t)| g_{0}(t) t^{2-d} d t .
$$


Proof. We introduce the operator $A_{\lambda, 0}^{D}$, which is associated with the quadratic form

$$
Q_{0}^{D}[f]:=\int_{0}^{\infty}\left(\left|f^{\prime}\right|^{2}+\lambda V(t)|f|^{2}\right) g_{0}(t) d t, \quad D\left(Q_{0}^{D}\right)=H_{0}^{1}\left(\mathbb{R}_{+}, g_{0}\right),
$$

where $H_{0}^{1}\left(\mathbb{R}_{+}, g_{0}\right):=\left\{f \in H^{1}\left(\mathbb{R}_{+}, g_{0}\right), f(0)=0\right\}$. First we observe that

$$
a t^{d-1} \leq g_{0}(t), \quad t \in \mathbb{R}_{+}
$$

for a suitable $a>0$. We can thus mimic the analysis of Section 3.1 and define the operator $\tilde{A}_{\lambda}$ acting in $L^{2}\left(\mathbb{R}_{+}, t^{d-1}\right)$ associated with the quadratic form

$$
\tilde{Q}[f]=\int_{0}^{\infty}\left(\left|f^{\prime}\right|^{2}+\lambda \tilde{V}(t)|f|^{2}\right) t^{d-1} d t, \quad f \in D(Q)
$$

where $D(Q)=H_{0}^{1}\left(\left(\mathbb{R}_{+}\right), t^{d-1}\right)$ and $\tilde{V}(t):=\frac{g_{0}(t)}{a t^{d-1}} V(t)$. Repeating the arguments of Section 3.1 we claim that

$$
N_{-}\left(A_{\lambda, 0}^{D}\right) \leq N_{-}\left(\tilde{A}_{\lambda}\right)
$$

and that $\tilde{A}_{\lambda}$ is unitarily equivalent to $\tilde{B}_{\lambda}$ by means of the transformation $\tilde{U} f(t)=t^{(d-1) / 2} f(t)$, which maps $L^{2}\left(\mathbb{R}_{+}, t^{d-1}\right)$ unitarily onto $L^{2}\left(\mathbb{R}_{+}\right)$. Since the co-dimension of $H_{0}^{1}\left(\mathbb{R}_{+}, g_{0}\right)$ in $H^{1}\left(\mathbb{R}_{+}, g_{0}\right)$ is equal to one, the variational principle gives

$$
N_{-}\left(A_{\lambda, 0}\right) \leq 1+N_{-}\left(A_{\lambda, 0}^{D}\right) \leq 1+N_{-}\left(\tilde{A}_{\lambda}\right)=1+N_{-}\left(\tilde{B}_{\lambda}\right) .
$$

Application of Proposition 1 with $\tilde{K}(d)=a K(d)$ concludes the proof.

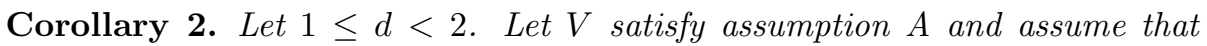
$\int_{0}^{\infty} t|V(t)| d t<\infty$. Then there exists $\lambda_{c}>0$, so that for $\lambda \in\left[0, \lambda_{c}\right]$ the discrete spectra of the operators $A_{\lambda, k}, k \geq 1$ are empty. In particular we have

$$
\sigma_{d}\left(A_{\lambda}\right)=\sigma_{d}\left(A_{\lambda, 0}\right), \quad 0 \leq \lambda \leq \lambda_{c}
$$

where the multiplicities of the eigenvalues are taken into account.

Proof. Let $k \geq 1$ be fixed. In view of Lemma 1 it suffices to show that the discrete spectrum of the operator $B_{\lambda, k}^{-}$is empty provided $\lambda$ is small enough. Since $(d-1)(d-3) \leq 0$, the following inequality holds true in the sense of quadratic forms:

$$
B_{\lambda, k}^{-} \geq \mathcal{B}_{\lambda, k}:=-\frac{d^{2}}{d t^{2}}+\frac{(d-1)(d-3)}{4\left(t-t_{k}\right)^{2}}+\lambda V_{k}^{-}(t),
$$

where the domain of $\mathcal{B}_{\lambda, k}$ coincides with that of $B_{\lambda, k}^{-}$given in Lemma $\square$ A simple translation $s=t-t_{k}$ then shows that $\mathcal{B}_{\lambda, k}$ is unitarily equivalent to the operator

$$
-\frac{d^{2}}{d s^{2}}+\frac{(d-1)(d-3)}{4 s^{2}}+\lambda V_{k}^{-}\left(s+t_{k}\right)
$$


acting in $L^{2}\left(\mathbb{R}_{+}\right)$with Dirichlet boundary condition at zero. To finish the proof it thus remains to apply Proposition 1 with

$$
\tilde{V}(s)=\frac{g_{k}\left(s+t_{k}\right)}{g_{k}^{-}\left(s+t_{k}\right)} V\left(s+t_{k}\right), \quad s \in[0, \infty)
$$

and take $\lambda$ small enough, such that $N_{-}\left(\mathcal{B}_{\lambda, k}\right)=0$.

\section{$5 \quad$ Weak coupling}

\subsection{The case $1 \leq d<2$}

In this section we will show that if $d \in[1,2)$ and $V$ is attractive in certain sense, then the operator $A_{\lambda}$ possesses at least one negative eigenvalue for any $\lambda>0$. Since for small values of $\lambda$ the discrete spectra of $A_{\lambda}$ and $A_{\lambda, 0}$ coincide, see Corollary 2 we will focus on the operator $A_{\lambda, 0}$ only. More exactly, in view of (8), we will study the operators $B_{\lambda, 0}^{ \pm}$. Clearly we have

$$
B_{\lambda, 0}^{ \pm}=B_{0}+\lambda V_{0}^{ \pm}, \quad B_{0}:=-\frac{d^{2}}{d t^{2}}+\frac{(d-1)(d-3)}{4(1+t)^{2}},
$$

with the boundary condition $v^{\prime}(0)=\frac{d-1}{2} v(0)$. Note that, by Lemma 1 the operator $B_{0}$ is non-negative. We shall first calculate the Green function of $B_{0}$ at a point $-\kappa^{2}, \kappa>0$, using the Sturm-Liouville theory again. In the same manner as in the previous section we obtain

$$
G\left(t, t^{\prime}, \kappa\right):=\left\{\begin{array}{ll}
\frac{\pi}{4 i \beta(\kappa)} v_{1}(t, \kappa) v_{2}\left(t^{\prime}, \kappa\right) & t \geq t^{\prime} \\
\frac{\pi}{4 i \beta(\kappa)} v_{1}\left(t^{\prime}, \kappa\right) v_{2}(t, \kappa) & t<t^{\prime}
\end{array},\right.
$$

where

$$
\begin{aligned}
v_{1}(t, \kappa) & =\sqrt{1+t} H_{\nu}^{(1)}(i \kappa(1+t)) \\
v_{2}(t, \kappa) & =\sqrt{1+t}\left(H_{\nu}^{(1)}(i \kappa(1+t))-\beta(\kappa) H_{\nu}^{(2)}(i \kappa(1+t))\right), \\
\beta(\kappa) & =\frac{H_{\nu-1}^{(1)}(i \kappa)}{H_{\nu-1}^{(2)}(i \kappa)} .
\end{aligned}
$$

Consider a function $W$ which satisfies assumption A According to the Birman-Schwinger principle the operator $B_{0}+\lambda W$ has an eigenvalue $-\kappa^{2}$ if and only if the operator

$$
K(\kappa):=|W|^{1 / 2}\left(B_{0}+\kappa^{2}\right)^{-1} W^{1 / 2}
$$

has eigenvalue $-\lambda^{-1}$. The integral kernel of $K(\kappa)$ is equal to

$$
K\left(t, t^{\prime}, \kappa\right)=|W(t)|^{1 / 2} G\left(t, t^{\prime}, \kappa\right)\left(W\left(t^{\prime}\right)\right)^{1 / 2} .
$$


We will use the decomposition

$$
K\left(t, t^{\prime}, \kappa\right)=L\left(t, t^{\prime}, \kappa\right)+M\left(t, t^{\prime}, \kappa\right),
$$

with

$$
L\left(t, t^{\prime}, \kappa\right):=\frac{\pi 2^{2 \nu-1} \kappa^{-2 \nu}}{(\Gamma(1-\nu))^{2} \sin (\nu \pi)}|W(t)|^{1 / 2}\left[(1+t)\left(1+t^{\prime}\right)\right]^{-\nu+\frac{1}{2}} W\left(t^{\prime}\right)^{1 / 2},
$$

and denote by $L(\kappa)$ and $M(\kappa)$ the integral operators with the kernels $L\left(t, t^{\prime}, \kappa\right)$ and $M\left(t, t^{\prime}, \kappa\right)$ respectively. Furthermore, we denote by $M(0)$ the integral operator with the kernel

$$
M\left(t, t^{\prime}, 0\right):=C_{M}(\nu)\left(|W(t)| W\left(t^{\prime}\right)(1+t)\left(1+t^{\prime}\right)\right)^{\frac{1}{2}}\left(\frac{1+t}{1+t^{\prime}}\right)^{\nu \operatorname{sign}\left(t-t^{\prime}\right)}
$$

where

$$
C_{M}(\nu):=-\frac{\pi}{2 \sin (\nu \pi) \Gamma(1-\nu) \Gamma(1+\nu)} .
$$

Lemma 2 in the Appendix says that $M(\kappa)$ converges in the Hilbert-Schmidt norm to the operator $M(0)$ as $\kappa \rightarrow 0$, provided $W$ decays fast enough at infinity. This allows us to prove

Theorem 2. Assume that $W$ satisfies $₫$ and that $\int_{0}^{\infty}(1+t)^{3-d}|W(t)| d t<\infty$, where $1 \leq d<2$. Then the following statements hold true.

(a) If

$$
\int_{0}^{\infty} W(t)(1+t)^{d-1} d t<0
$$

then the operator $B_{0}+\lambda W$ has at least one negative eigenvalue for all $\lambda>0$. For $\lambda$ small enough this eigenvalue, denoted by $E(\lambda)$, is unique and satisfies

$$
(E(\lambda))^{\frac{2-d}{2}}=C(\nu)\left(\lambda \int_{0}^{\infty} W(t)(1+t)^{d-1} d t+\mathcal{O}\left(\lambda^{2}\right)\right)
$$

where

$$
C(\nu)=\frac{\pi 2^{2 \nu-1}}{(\Gamma(1-\nu))^{2} \sin (\nu \pi)}
$$

(b) If

$$
\int_{0}^{\infty} W(t)(1+t)^{d-1} d t>0,
$$

then the operator $B_{0}+\lambda W$ has no negative eigenvalues for $\lambda$ positive and small enough. 
Proof. Part (a).

The operator $B_{0}+\lambda W$ has eigenvalue $E=-\kappa^{2}$ if and only if the operator

$$
\lambda K(\kappa)=\lambda M(\kappa)+\lambda L(\kappa)
$$

has an eigenvalue -1 for certain $\kappa(\lambda)$. On the other hand, Lemma 1 and (7) imply that

$$
N_{-}\left(B_{0}+\lambda \frac{g_{0}}{g_{0}^{+}} V\right) \leq N_{-}\left(A_{\lambda, 0}\right) .
$$

The uniqueness of $E$, and so of $\kappa(\lambda)$, for $\lambda$ small enough thus follows from (19) by taking $V=\frac{g_{0}^{+}}{g_{0}} W$. Next we note that by Lemma 2 for $\lambda$ small we have $\lambda\|M(\kappa)\|<1$ and

$$
(I+\lambda K(\kappa))^{-1}=\left[I+\lambda(I+\lambda M(\kappa))^{-1} L(\kappa)\right]^{-1}(I+\lambda M(\kappa))^{-1} .
$$

So $\lambda K(\kappa)$ has an eigenvalue -1 if and only if $\lambda(I+\lambda M(\kappa))^{-1} L(\kappa)$ has an eigenvalue -1 . Since $\lambda(I+\lambda M(\kappa))^{-1} L(\kappa)$ is of rank one we get the equation for $\kappa(\lambda)$ in the form

$$
\operatorname{tr}\left(\lambda\left(I+\lambda M(\kappa(\lambda))^{-1} L(\kappa(\lambda))\right)=-1 .\right.
$$

Using the decomposition

$$
(I+\lambda M(\kappa))^{-1}=I-\lambda M(0)-\lambda(M(\kappa)-M(0))+\lambda^{2} M^{2}(\kappa)(I+\lambda M(\kappa))^{-1}
$$

we obtain

$$
\begin{aligned}
& \operatorname{tr}\left(\lambda(I+\lambda M(\kappa))^{-1} L(\kappa)\right) \\
& =\lambda C(\nu) \kappa^{-2 \nu}\left(|W(t)|^{1 / 2}(1+t)^{-\nu+\frac{1}{2}},(I+\lambda M(\kappa))^{-1} W(t)^{1 / 2}(1+t)^{-\nu+\frac{1}{2}}\right) \\
& =C(\nu) \kappa^{-2 \nu}\left(\lambda \int_{0}^{\infty} W(t)(1+t)^{d-1} d t+\mathcal{O}\left(\lambda^{2}\right)\right) .
\end{aligned}
$$

It thus follows from (24) that

$$
E^{\nu}(\lambda)=-\kappa^{2 \nu}(\lambda)=C(\nu)\left(\lambda \int_{0}^{\infty} W(t)(1+t)^{d-1} d t+\mathcal{O}\left(\lambda^{2}\right)\right) .
$$

To finish the proof of the part $(a)$ of the Theorem we mimic the argument used in [9] and notice that if $\left(\varphi,\left(B_{0}+\lambda W\right) \varphi\right)<0$, then $(\varphi, W \varphi)<0$, since $B_{0}$ is non-negative, and therefore $\left(\varphi,\left(B_{0}+\tilde{\lambda} W\right) \varphi\right)<0$ if $\lambda<\tilde{\lambda}$. So if $B_{0}+\lambda W$ has a negative eigenvalue for $\lambda$ small enough, then, by the variational principle, it has at least one negative eigenvalue for all $\lambda$ positive.

Part $(b)$. From the proof of part $(a)$ it can be easily seen that if

$$
\int_{0}^{\infty} W(t)(1+t)^{d-1} d t>0
$$

then $\operatorname{tr}\left(\lambda(I+\lambda M(\kappa))^{-1} L(\kappa)\right)$ is positive for $\lambda$ small and therefore $K(\kappa)$ cannot have an eigenvalue -1 . 
Remark 3. Note that if

$$
W_{0}:=\int_{\mathbb{R}_{+}^{2}} W(t) W\left(t^{\prime}\right)(1+t)^{1-\nu}\left(1+t^{\prime}\right)^{1-\nu}\left(\frac{1+t}{1+t^{\prime}}\right)^{\nu \operatorname{sign}\left(t-t^{\prime}\right)} d t d t^{\prime}<0,
$$

then the operator $B_{0}+\lambda W$ has a negative eigenvalue for $\lambda$ small, positive or negative, also in the critical case when

$$
\int_{0}^{\infty} W(t)(1+t)^{d-1} d t=0 .
$$

Moreover, it follows from the proof of Theorem 2 that this eigenvalue then satisfies

$$
E^{\nu}(\lambda)=C(\nu)\left(-\lambda^{2} C_{M}(\nu) W_{0}+o\left(\lambda^{2}\right)\right), \quad \lambda \rightarrow 0 .
$$

As an immediate consequence of Theorem 2 and inequalities (8) we get

Theorem 3. Let $V$ satisfy assumption $\square$ and let $\int_{0}^{\infty}(1+t)^{3-d}|V(t)| d t<\infty$, where $1 \leq d<2$. Then the following statements hold true.

(a) If

$$
\int_{0}^{\infty} V(t) g_{0}(t) d t=\int_{\Gamma} V(|x|) d x<0,
$$

then the operator $A_{\lambda}$ has at least one negative eigenvalue $E_{1,0}(\lambda)$ for all $\lambda>0$. For $\lambda$ small enough this eigenvalue is unique and satisfies

$$
C_{1}\left|\lambda \int_{\Gamma} V(|x|) d x\right|^{\frac{2}{2-d}} \leq\left|E_{1,0}(\lambda)\right| \leq C_{2}\left|\lambda \int_{\Gamma} V(|x|) d x\right|^{\frac{2}{2-d}}
$$

for suitable positive constants $C_{1}$ and $C_{2}$.

(b) If

$$
\int_{0}^{\infty} V(t) g_{0}(t) d t=\int_{\Gamma} V(|x|) d x>0
$$

then the discrete spectrum of $A_{\lambda}$ is empty for $\lambda$ positive and small enough.

Proof. Part (a). From (8) we get

$$
E_{1,0}^{-}(\lambda) \leq E_{1,0}(\lambda) \leq E_{1,0}^{+}(\lambda) .
$$

Moreover, by Lemma $1 E_{1,0}^{ \pm}(\lambda)$ are the lowest eigenvalues of operators $B_{\lambda, 0}^{ \pm}$. The existence and uniqueness of $E_{1,0}$ thus follows from part (a) of Theorem 2 applied with $W(t)=V_{0}^{+}(t)$ and $W(t)=V_{0}^{-}(t)$ respectively. At the same time, equation (23) implies (27).

Similarly, part (b) of the statement follows immediately from Lemma 1 and part (b) of Theorem 2 applied with $W(t)=V_{0}^{-}(t)$. 
Remark 4. We note that the strong coupling behavior of $A_{\lambda}$ is, on the contrary to (27), typically one-dimensional, i.e. determined by the local dimension of $\Gamma$. Namely, if $V$ is continuous and compactly supported, then the standard Dirichlet-Neumann bracketing technique shows that the Weyl asymptotic formula

$$
\lim _{\lambda \rightarrow \infty} \lambda^{-\gamma-\frac{1}{2}} \sum_{j}\left|E_{j}\right|^{\gamma}=L_{\gamma, 1}^{c l} \int_{\Gamma}|V|^{\gamma+\frac{1}{2}} d x, \quad \gamma \geq 0
$$

holds true, where $E_{j}$ are the negative eigenvalues of $A_{\lambda}$ and $L_{\gamma, 1}^{c l}=\frac{\Gamma(\gamma+1)}{2 \sqrt{\pi} \Gamma(\gamma+3 / 2)}$.

\subsection{The case $d=2$}

For $d=2$ one can mimic the above procedure replacing the Hankel functions $H_{\nu}^{(1,2)}$ by $H_{0}^{(1,2)}$. The latter have a logarithmic singularity at zero and therefore it turns out that the lowest eigenvalue of $A_{\lambda}$ then converges to zero exponentially fast. Indeed, here instead of (23) one obtains

$$
E(\lambda) \sim-e^{-\lambda^{-1}},
$$

as for the two-dimensional Schrödinger operator, see [9]. Since the analysis of this case is completely analogous to the previous one, we skip it.

\subsection{The case $d>2$}

Now it remains to show that for $d>2$ and $\lambda$ small enough the discrete spectrum of $A_{\lambda}$, which in this case coincides with that of $A_{\lambda, 0}$, remains empty. Since the discrete spectrum of $A_{\lambda, 0}$ might only consist of negative eigenvalues, see (13), it suffices to prove

Proposition 2. If $d>2$ and $V \in L^{\infty}\left(\mathbb{R}_{+}\right) \cap L^{d / 2}\left(\mathbb{R}_{+}, g_{0}\right)$, then there exists $\lambda_{0}>0$ such that the operator $A_{\lambda, 0}$ is non-negative for all $\lambda \in\left[0, \lambda_{0}\right]$.

Proof. Consider a function $f \in D\left(Q_{0}\right)$. Since $f \in H^{1}\left(\mathbb{R}_{+}\right)$, which is continuously embedded in $L^{\infty}\left(\mathbb{R}_{+}\right)$, it follows that $f \rightarrow 0$ at infinity and we can write

$$
f(t)=-\int_{t}^{\infty} f^{\prime}(s) d s .
$$

In view of (44) we have $g_{0}^{-1} \in L^{1}\left(\mathbb{R}_{+}\right)$. Using Cauchy-Schwarz inequality we thus find out that for any $q \geq q_{0}$, where $\frac{1}{q_{0}}+\frac{1}{d}=\frac{1}{2}$, the following estimate holds true

$$
\begin{aligned}
& \left(\int_{0}^{\infty}|f(t)|^{q} g_{0}(t) d t\right)^{\frac{1}{q}} \leq\left(\int_{0}^{\infty}\left(\int_{t}^{\infty}\left|f^{\prime}(s)\right| d s\right)^{q} g_{0}(t) d t\right)^{\frac{1}{q}} \\
& \leq\left(\int_{0}^{\infty}\left(\int_{t}^{\infty}\left|f^{\prime}(s)\right|^{2} g_{0}(s) d s\right)^{\frac{q}{2}}\left(\int_{t}^{\infty} \frac{d s}{g_{0}(s)}\right)^{\frac{q}{2}} g_{0}(t) d t\right)^{\frac{1}{q}} \\
& \leq C(q)\left(\int_{0}^{\infty}\left|f^{\prime}(s)\right|^{2} g_{0}(s) d s\right)^{\frac{1}{2}}
\end{aligned}
$$


with a constant $C(q)$ independent of $f$. The Hölder inequality and (28) then give

$$
\begin{aligned}
\int_{0}^{\infty}|V||f|^{2} g_{0}(t) d t & \leq\left(\int_{0}^{\infty}|V|^{d / 2} g_{0}(t) d t\right)^{\frac{2}{d}}\left(\int_{0}^{\infty}|f|^{q_{0}} g_{0}(t) d t\right)^{\frac{2}{q_{0}}} \\
& \leq C^{2}\left(q_{0}\right) \int_{0}^{\infty}\left|f^{\prime}\right|^{2} g_{0}(t) d t\left(\int_{0}^{\infty}|V|^{d / 2} g_{0}(t) d t\right)^{\frac{2}{d}}
\end{aligned}
$$

which implies

$$
Q_{0}[f] \geq \int_{0}^{\infty}\left|f^{\prime}\right|^{2} g_{0}(t) d t\left[1-\lambda C^{2}\left(q_{0}\right)\left(\int_{0}^{\infty}|V|^{d / 2} g_{0}(t) d t\right)^{\frac{2}{d}}\right] .
$$

To finish the proof it suffices to take $\lambda$ small enough so that $Q_{0}[f] \geq 0$.

\section{Appendix}

Lemma 2. Let $W$ be bounded and assume that $\int_{0}^{\infty}(1+t)^{1+2 \nu}|W(t)| d t<\infty$. Then $M(\kappa)$ converges in the Hilbert-Schmidt norm to the operator $M(0)$ as $\kappa \rightarrow 0$.

Proof. We first notice that $M(0)$ is Hilbert-Schmidt, since

$$
\int_{0}^{\infty} \int_{0}^{\infty}\left|M\left(t, t^{\prime}, 0\right)\right|^{2} d t d t^{\prime}<\infty
$$

by assumption. We will also need the asymptotic behavior of the Bessel functions with purely imaginary argument near zero:

$$
J_{\nu}(i \kappa(1+t))=e^{i \pi \nu / 2} I_{\nu}(\kappa(1+t)) \sim e^{i \pi \nu / 2} \frac{\kappa^{\nu}(1+t)^{\nu}}{2^{\nu} \Gamma(\nu+1)}, \quad \kappa(1+t) \rightarrow 0,
$$

see [1, 12. From the definition of Hankel's functions we thus get

$$
\beta(\kappa)=\frac{J_{1-\nu}(i \kappa)-e^{i(1-\nu) \pi} J_{\nu-1}(i \kappa)}{e^{i(\nu-1) \pi} J_{\nu-1}(i \kappa)-J_{1-\nu}(i \kappa)} \rightarrow-e^{-2 i \nu \pi}, \quad \kappa \rightarrow 0 .
$$

This together with the asymptotics (29) implies

$$
\lim _{\kappa \rightarrow 0} M\left(t, t^{\prime}, \kappa\right)=M\left(t, t^{\prime}, 0\right) .
$$

Now using the asymptotic behavior of Hankel's functions at infinity, [1], we find out that

$G\left(t, t^{\prime}, \kappa\right) \sim\left((1+t)\left(1+t^{\prime}\right)\right)^{1 / 2} \frac{e^{-\kappa\left(t+t^{\prime}\right)}-\beta(\kappa) e^{-\kappa\left|t-t^{\prime}\right|}}{\kappa(1+t)\left(1+t^{\prime}\right)^{1 / 2}}, \quad \kappa^{2}(1+t)\left(1+t^{\prime}\right) \rightarrow \infty$.

Since $|\beta(\kappa)|$ is bounded, we obtain the following estimates. 
For $\kappa^{2}(1+t)\left(1+t^{\prime}\right) \geq 1$ :

$$
\left|K\left(t, t^{\prime}, \kappa\right)\right|,\left|L\left(t, t^{\prime}, \kappa\right)\right| \leq C\left|W\left(t^{\prime}\right) W(t)(1+t)\left(1+t^{\prime}\right)\right|^{1 / 2} .
$$

For $\kappa^{2}(1+t)\left(1+t^{\prime}\right)<1$ :

$$
\left|M\left(t, t^{\prime}, \kappa\right)\right| \leq C^{\prime}\left|W\left(t^{\prime}\right) W(t)\right|\left[1+\left((1+t)\left(1+t^{\prime}\right)\right)^{\nu+\frac{1}{2}}\right],
$$

where we have used (30). Note that the constants $C$ and $C^{\prime}$ may be chosen independent of $\kappa$, which enables us to employ the Lebesgue dominated convergence theorem to conclude that

$$
\lim _{\kappa \rightarrow 0} \int_{\mathbb{R}_{+}^{2}}\left|M\left(t, t^{\prime}, \kappa\right)-M\left(t, t^{\prime}, 0\right)\right|^{2} d t d t^{\prime}=0 .
$$

\section{Acknowledgement}

The work has been partially supported by the Czech Academy of Sciences and by DAAD within the project D-CZ 5/05-06.

\section{References}

[1] M. Abramowitz and I.A. Stegun: Handbook of Mathematical Functions, National Bureau of Standards (1964).

[2] V. Bargmann: On the number of bound states in a central field of force, Proc. Nat. Acad. Sci. U.S.A. 38 (1952) 961-966.

[3] M.S. Birman and M.Z. Solomyak: Schrödinger Operator. Estimates for number of bound states as function-theoretical problem, Amer. Math. Soc. Transl. (2) Vol. 150 (1992).

[4] R. Carlson, Nonclassical Sturm-Liouville problems and Schrödinger operators on radial trees, Elect. J. Diff. Equation 71 (2000).

[5] P. Exner: Weakly Coupled States on Branching Graphs, Letters in Math. Phys. 38 (1996) 313-320.

[6] K. Naimark and M. Solomyak, Geometry of the Sobolev spaces on the regular trees and Hardy's inequalities, Russian Journal of Math. Phys. 8 (2001) 322-335.

[7] K. Naimark and M. Solomyak, Eigenvalue estimates for the weighted Laplacian on metric trees, Proc. London Math. Soc. 80 (2000) 690-724. 
[8] M. Reed, B. Simon, Methods of Modern Mathematical Physics, IV, Academic press, New York (1978).

[9] B. Simon, The Bound State of Weakly Coupled Schrödinger Operators in One and Two Dimensions, Ann. of Physics 97 (1976) 279-288.

[10] A. Sobolev and M. Solomyak, Schrödinger operators on homogeneous metric trees: spectrum in gaps, Rev. Math. Phys. 14 (2002) 421-467.

[11] M. Solomyak, On the spectrum of the Laplacian on metric trees, Waves in Rand. Media 14 (2004) S155-S171.

[12] G. N. Watson, A treatise on the theory of Bessel functions, Cambridge University Press (1958). 